\title{
Research on the Aesthetic Feature of Material in Industrial Product Design
}

\author{
Lili Sun \\ School of Design and Art \\ Harbin University of Commerce \\ Harbin, China
}

\begin{abstract}
Material is the basic problem needed to be solved by the modern industrial product designer. With the continuous improvement to product emotion and aesthetic demand of people, product designers should take the material as aesthetic object of main body, and comprehensively review and process it with unique artistic creation ability. This paper respectively discussed the aesthetic feature of material in product design from the three aspects of the visual aesthetic feeling of the materials, touch aesthetic experience and the cultural aesthetic connotation.
\end{abstract}

Keywords—product design; material; aesthetic feature

\section{INTRODUCTION}

Materials, as an essential part of all human activity of creation for material basis, reflects the development level of science and technology at a certain extent, at the same time embodies the social, economic value and the cultural spirit of the age. There is discussion in Kaogongji that "When the day is in right time, the earth has right air, the craftsman has skillful ability and the material is beautiful, then the good products can be created.", the "material is beautiful" emphasizes the aesthetic characteristics of materials in the activity of creation, which not only includes the appreciation of the beauty to the matter properties of the material, also contains the spirit and cultural connotation displayed under the harmonious development between the material and human civilization. In the face of the problems that how to choose appropriate materials in industrial product design, how to bring out the best in each other between the material and product elements, and reflects the unique aesthetic taste and cultural connotation of product, need to understand the aesthetic features of the material from multiple perspectives comprehensively in modern product design.

\section{Visual AESTHETIC EMOTION OF THE MATERIAL}

The visual characteristic of material includes material color, luster, texture, transparency, etc., is a kind of feeling and impression gotten from the brain comprehensive treatment to the material touched by visual sense organs. The visual stimulation account about for more than $80 \%$ of all external stimuli accepted by the human brain according to scientific survey, human perceive the world, understand the world through human eyes and produce a series of physiological and psychological reaction after the stimulation in visual organs, which produce different emotional consciousness, therefore, visual characteristic of material contains two aspects, namely visual physical characteristic and visual psychological characteristic.

Visual characteristic of material mainly displays via the surface physical properties, the different surface color, luster, texture etc. will make people arise the visual and psychological differences. In color respect, it can make the products have different personality and influence people's emotional experience by using the material with a different hue, lightness and purity. Psychologists have proved through scientific experiments that the psychological effect of color produced by the different performance of human brain waves stimulated by different color, such as the reaction of brain waves are alert and impulsive under the red stimuli, and blue stimuli is relaxed and calm, and the lightness, bright color gives a person the feeling of lightness, while the dark color makes him feel heavy. Wood, for example, the ones with high brightness, good luster, such as basswood, birch, cedar and so on generally give a person the lively, natural and light sense, while the taxus chinensis, red sandalwood etc. with low lightness often provide a person the luxury, deep, simple but elegant and dignified feeling. In terms of shine and texture, the transparent, smooth and bright visual features of glass material make the person produce the cold, romantic, light and delicate psychological characteristics. The hard, bright, reflective properties of metal can make the person get cold, strength and lively feeling, and the neat and cool feeling produced by the fine and smooth ceramic is different from the primitive simplicity, romance and natural feeling produced by the dark dumb and folds of the leather.

With the development of science and technology, more and more processing means provide the infinite possibility for designing materials on the vision. Different processing methods and technology of the skills can make a difference in appearance, and then the unique feeling feature is achieved. Such as the common processing technology, forging process can produce a very rich texture effect, or bold and vigorous or fine and beautiful, which have strong personality and manual beauty due to the completely kept emotional traces. Such as the good duplicating function of casting process can repeat the fine tendrils, rough rock, and can produce peculiar texture beauty by deliberately kept traces of welding. And the sand blasting process can produce 
different levels of rough surface for material, electroplating technology can change the surface properties of materials and get metallic luster effect. And process a kind of material with different processing methods can obtain different visual aesthetic feeling, such as after chrome plating treatment to the plastic surface, the appearance of it is delicate, dazzling and can be compared to metal luxurious. After grinding, etching, sandblasting and chemical etching processing, the glass can also produce the rough, yogon effects which are different from the original visual features. Under the driven of the technical processing progress and innovation in the material surface, the visual language expression on the surface of the modern product will become increasingly rich and varied.

\section{THE TOUCHING AESTHETIC EXPERIENCE OF THE MATERIAL}

Touch is a composite composed of sports feeling and the skin feeling. The sports feeling refers to the feeling of body movement and position of state; Skin feeling refers to identify the temperature of the objects, chemical and mechanical characteristics, which are generally divided into temperature, pressure, pain, etc. Touch ranks only second to the visual sensitivity for people know the things and the environment, which plays an very important role to develop the sensation and perception to the of people. The material will pass the physical properties of it via the stimulated surface receptors of the skin touched with the material during the products using process, and produce the sensory experience and the change at psychological level, such as porosity, density and hardness of material determines the quality of it, such as glass, ceramics, metal and so on, people will feel smooth and cold because of its close texture, high thermal conductivity, while the thermal conductivity of cotton fabric and wood is low due to its loose texture, which usually can make human body feel rough and warm.

Tactile aesthetic characteristics of the material will produce pleasure tactility which is embodied in the using process of our users, generally speaking, compare to the sticky, astringent and other unpleasant feelings, people are more likely to touch the delicate, soft, smooth and moist materials, for example, wood is a poor conductor of heat, heat preservation performance is good, warm tactile sense make it as the ideal material for furniture often contacted with the human body, biomass materials such as cotton and silk has an excellent bio-compatibility with human body and comfortable touch, therefore they become as the main fabric of dress design. Some of the new material also arises at the historic moment in order to meet the needs of people on touch beauty for material, such as a new type of silicone soft keyboard, the material brings a kind of the delicate \& moisten feeling like baby's skin, which improve the efficiency of the use and enjoyment; And some of the common material has also been cleverly misappropriate, for example, the wool knitting brings the cordial and familiar tactile has been cleverly used in the design of a kind of furniture coated by a knitted fabric made of the special thick wool yarn and coarse cotton wool, which brings the warm and comfortable feeling to users taking this material surface as seat in usage. The touch feature of material also embodies in improving the applicability of the product and efficiency in modern product design, such as during the design of handle for a variety of tools, the coarse grain process will be done on the material or choose anti-skid material such as rubber, leather and so on, for example, some hand-held tool handle is sprayed with high touch plastic paint, so that it is not easy to slide and feel comfortable.At the same time, the integrated application of a variety of different tactile materials make a product as a perfect combination of various materials with different skin feature, and texture, which not only satisfies the practical function of the product, also enriches product model-ling art language to meet the user's sensory experience and enhance the degree of psychological pleasure.

\section{The Culture Aesthetic Connotation of MATERIAL}

In the process of human social civilization, under the influence of various social factors, the inherent nature of physical properties and the emotion of material is often endowed with the connotation of history and culture, which make the material with rich cultural feature affect the design thinking and direction, then it is necessary that the choice of materials should be considered in the process of design, while the function and meaning on reflecting and influencing the culture of the material should be pay much more attention. Materials bring the unique physiological and psychological experience to people through their shape, texture, color and texture, with the closeness degree between the material and people, each kind of material has showed the unique temperament in the integration of factors such as history and geography, climate and so on, and then become as the art language containing the culture and the emotion. As all the material basis for the creation activity, each material has its corresponding era of cultural background, such as the painted pottery delegates the humans enter the civilization from the barbarian state of the new Stone Age, the bronze ware reflects the Shang and Zhou period with hierarchy in the slave society of the bronze ware, the gold and silver wares shows the due to the Wei Jin Southern and Northern Dynasties period with the incoming of Buddhist culture. The material bears the mission of carrying out the history and culture and inter-prate the unique spiritual world and humanistic care. Material is shouldering the historical and cultural mission and interpretation of the unique spiritual world and humanistic care.

In famous of its simplicity and elegance of Ming style furniture much choose hard wood with high quality such as red sandalwood, rosewood, wenge and so on, their material texture is solid, gloss is bloom, grain is exquisite soft and gentle elegant, which not only meets the using function, also embodies the rigid-flexible beauty. It both emphasizes the nature of furniture material, also pay attention to the perfect unification of artificial work, which integrate the "natural beauty" of the Taoist culture and the "humanistic beauty" of Confucian culture .Therefore, the high quality design not only means inspecting the material and accurately applying the characteristics of the materials, also lies in the 
understanding and digging the cultural connotation of the materials, and then carrying forward its essence. In the case of bamboo, bamboo is not only a kind of natural material with tenacity and lighter characteristics, but also contains the rich connotation of national tradition culture, which means longevity, good fortune, promoting to a higher position among the people; In the heart of letters, as one of the four gentleman, it has the noble character of tenacious vitality and unyielding feature and the charm of the elegant and free from vulgarity, which makes bamboo as the green environmental protection material for furniture and other products; Japan, with "similar" Chinese culture, taking bamboo as the material for the design of Zen household product, has incisively and vividly display the quiet, comfortable and ethereal Zen aesthetics charm via its contracted model-ling and fine detail. Historically, the evolution of materials directly influences the change of the artistic style, and leads the development of design culture and design style of this period to some extent. E.g., the coarse linen of Pre-qin period gives a person the sense with straightforward simplicity while the silk brocade of Sui and Tang dynasties provides the exquisite luxuriant to people; Material reflects their unique artistic temperament and affects the change of the concept of creation, different styles of clothes silently convey the simple sense of beauty, color and meaning given by material at different times, therefore, it is directly related to the artistic level and cultural value of product that to understand and dig the culture aesthetic connotation of the material.

People living in a variety of materials have become an inherent concept which roots in our ideological and cultural fields. Material and human is always an inseparable relationship, reference or imitate tend to become the most common form in the design in the face of a new material; Of course, there are some avant-courier dare to do innovation and application, so the performance of the new material is gradually mastered and continuously applied, which will also form new design trends, and then create the new design style and form of expression adapted to material .At the same time, the past form and style are gradually precipitation in the bold design experiment and under the screening of history, it is possible to dig up the much more potential of materials, use a much more mature material to encourage, guide and inspire designers to create, refine and sublimate to make it become another power to promote the development of design. This is obviously that a material acts on the design, design reacts upon material, and then material affects the design process.

An excellent designer will not only makes innovation of performance form and structure of products, also need to understand and explore the unique culture of each material. Design trend, under the influence of the design trend of though in the world, will shows diversified development trend; it has become inevitable that the social culture will gradually change to the pursuit of the nonphysical from the pursuit of material, which means when one enjoys the material life, pursuit the higher level of spiritual pleasure and cultural appreciation, the closeness and fusion to the nature and the more healthy and comfortable living style. Design must be considered the deeper levels including material culture. No matter the development and usage of natural materials done by human in the past, such as wood, bamboo rattan, clay and so on or the research and application to the new materials with the progress and development of science and technology, such as plastic, glass, alloys and so on, more and more material is applied to the design practice, each kind of material expresses the unique artistic style and charm with their own language. We should fully review and dig the aesthetic features of material, fully understand and reasonably treat the material in the product design, and adopt the properties and the artistic expression of each material at its the most suitable and ideal state, so that make the material not only as the material basis for the creation activity, at the same time as the carrier for creating aesthetic interest and expressing the emotion to really meet people's spiritual demand a higher level.

\section{REFERENCES}

[1] Biao Xu. The Way of becoming a Useful Thing or Useful Person [M] Jiangsu Arts Press, 2008

[2] Yixing Liu, Haipeng Yu, Rongjun Zhao. Science of Wood Environment [M]. Science Press,2007

[3] Guorong Wu, Yonggang Meng. Application of Material Feeling Feature in Product Design [J]. Packing Project 2006 (12):310-312

[4] Wei Xiang. Study of Non-matter Feature of Material in Product Design [J] Decoration. 2009 (10): 90-91

[5] Minglei Sun. Research on Traditional Aesthetic Connotation Embodied by Ming Style Furniture [D] Dongbei Forest University, 2005. 